\title{
Mental Health Multivariate Analysis for University Students with Left behind Experience
}

\author{
Cheng Yilin ${ }^{1}$ and Liu Zhiqiang ${ }^{2}$ \\ ${ }^{1,2}$ Xinzhou Teachers University, Shanxi, China \\ ${ }^{1}$ chengyilin@xztu.edu.cn
}

\begin{abstract}
To understand the mental health status of "university students with left-behind experience" in universities and universities, its influencing factors are analyzed, provide countermeasures to improve the mental health of "university students with left-behind experience", and provide references for the prevention of mental health problems of left-behind children in the future. 1,650 university students from three universities in a certain city were surveyed using the SCL90 scale and stratified cluster sampling method. The data was analyzed statistically using SAS9.1 statistical software. Multivariate analysis shows that the four variables of interpersonal relationship factor, hostility factor, compulsive factor, and teacher-student relationship are influential Main factors affecting the mental health of university students with left-behind experience. The survey showed that the left-behind population had a high positive rate of psychological problems, suggesting that left-behind experience is the main factor affecting their mental health. Gender, university type, and birthplace also have a certain impact on 10 factors, suggesting that future university students will have psychological education. Attention should be paid to this group of university students with left-behind experience, and to explore the impact of left-behind background on their mental health and improve their health.
\end{abstract}

Keywords: Left-behind, University student, Mental health, Social support

\section{Introduction}

Since the 1980s, with the continuous acceleration of China's reform and opening up and urbanization process, the surplus rural labour force has been transferred to cities on a large scale. Some of these "left-behind children" born in the late 1980s and early 1990s were fortunate to continue their studies and become university students with left-behind experiences. University students who have the left-behind experience, that is, those students whose parents or both have been away from their hometown for a long time before going to university, go out to work, do business, or study, and have separated from them for one year or more [1][2]. This research investigates the mental health status of university students with left-behind experience by investigating university students with left-behind experience in three types of universities in a city, analyzes its influencing factors, and proposes targeted interventions for mental health education of university students with left-behind experience [3][4]. It also proposes targeted interventions for the mental health problems of left-behind children in the future.

Article history:

Received (September 2, 2020), Review Result (October 8, 2020), Accepted (November 12, 2020)

Print ISSN: 2207-5380, eISSN: 2207-290X AJEMR

Copyright (C) 2020 Global Vision Press (GV Press) 


\section{Research object and method}

Taking a coastal city in Shandong Province as a research point, the stratified cluster sampling method was used to select the university students in the three universities in the city for investigation. The three universities were comprehensive universities, normal universities, and medical universities. For some freshmen to seniors of majors of each university, anonymous questionnaire surveys are carried out in class units, with uniformly trained professionals serving as investigators. The survey process uses uniform methods and guidance questionnaires to be distributed uniformly, answering on the spot, unified recovery, and verification.

A total of 1,650 questionnaires were distributed, and 1,605 valid questionnaires were recovered, with an efficiency of $97.27 \%$. Among them, 312 university students had the leftbehind experience, with a positive rate of $19.44 \%$. The SCL-90 symptom self-assessment scale is used. SCL-90 was compiled by the famous American Psychologist Derogates. 90 evaluation items, each item is estimated by the patient from none (1) to extreme (5). The scale includes somatization, strong pursuit syndrome, interpersonal relationships, depression, anxiety, hostile terror, paranoia, psychosis, and other 10 symptom dimensions or factors. Double data entry, collation, and logical error detection of data were used for statistical analysis through Epidata software, statistical analysis using SAS9.1 statistical software, analysis and comparison of factors affecting mental health using t-test, rank-sum test.

\section{Result}

\subsection{Population distribution}

1605 university students from three different types of universities (comprehensive, normal, medical) were selected, of which 312 students with left-behind experience", the positive rate was $19.44 \%$. the positive rate for comprehensive universities was $25.93 \%$, the positive rate for normal universities was $17.67 \%$, and the positive rate for medical universities was $17.01 \%$. Among the 312 university students with left-behind experience, males and females account for $39.7 \%$ and $60.3 \%$. $79.8 \%$ of them are from rural areas, and $64.7 \%$ are non-only children; from the perspective of grades, most students are from lower grades, of which freshmen account for $34.6 \%$, sophomores and juniors account for $26.6 \%, 29.5 \%$. the proportion of senior students is relatively small, accounting for $9.3 \%$, which is related to the choice of the survey object.

\subsection{Left-behind background and mental health status}

\subsubsection{Background of left-behind research}

This study set up several projects for left-behind students' left-behind experience and investigated the self-harmony and evaluation, emotional and social support of left-behind students.

From the perspective of self-harmony and evaluation, $38.5 \%$ of the 312 surveyed university students with left-behind experience think that their left-behind experience has changed their character. $66.7 \%$ of them think that this character change is positive. Only $33.3 \%$ of students think it's negative. From the experience of left-behind university students who care about the surrounding students, $22.8 \%$ think their neighbours, relatives, friends, teachers, and classmates care about them, and $51.6 \%$ think they are more negative concerned 
about themselves, $25.6 \%$ think they are little, unconcerned or indifferent to them. From the perspective of emotional and social support in university, 53.5\% think they have a normal relationship with teachers and students in the university. 9\% of students think that the relationship between teachers and students is not good or even bad. From the "Do you have many friends at university" project, 53.5\% of students think they have ordinary friends in university, $34.6 \%$ think they have many friends in university, and $11.8 \%$ of students think that they have few or even no friends in university, and $20.8 \%$ of them express they have no close friends in university. In "Who do you usually have problems communicating with?", On $63.8 \%$ of students choose to classmates or friends, $23.7 \%$ of students choose to talk to their parents, while only $1.6 \%$ choose to talk to a counsellor teacher, himself a man keep our noses to solve accounted for $7.1 \%$

\subsubsection{Mental health status of university students with left-behind experience}

Among the left-behind students, the average score of the evaluation factors of the mental health scale was 153.86 points, and the number of students with a factor score of more than 160 was 111 , accounting for $35.58 \%$ of the left-behind students. The test students may have some degree of psychological disorder. The total score of the factor was divided by the number of questions in the factor to calculate the average score of each factor. The average score of the obsessive-compulsive disorder factor is the highest at 1.95 , followed by the interpersonal relationship factor, depression factor, and hostility factor. Combined with the criteria for determining the positive rate of the scale, the top three positive detection rates of the left-behind population are the compulsive factor, interpersonal factor, and hostile factor.

\subsubsection{Comparison of the mental health status of university students with left-behind experience}

(1) Comparing the psychological status of university students with left-behind experience and those without.

In this study, the differences between the 10 factors of SCL-90 of university students with left-behind experience and non-left-behind students were compared. From the comparison of the ten factors of somatization, obsessive-compulsive state, interpersonal relationships, depression, banana worry, hostility, terror, paranoia, psychosis, and others (reflecting sleep and diet). The differences between the factors of the two populations are statistically significant Significance (all P values are less than 0.01), suggesting that "left-behind" is the influencing factor of university students' mental health, and the scores of various factors of university students with left-behind experience are higher than those of university students with no left-behind experience.

(2) Comparison of the factors influencing the psychological health of university students with left-behind experience.

This study compares the differences between university types, genders, and student origins that affect the mental health of university students with left-behind experience. From the perspective of university type, the differences between the three factors are statistically significant $(\mathrm{P}<0.05)$. Among them, the three factors of somatization, anxiety, and fear are the highest in medical universities. For the other factors, all-comprehensive universities have the highest scores. From the comparison of the scores of different factors between the genders, the differences between the genders of each factor are statistically significant. The average score of each factor is less than 2 points, and the boy's scores are both higher than girls as 
shown in [Table 1]. However, from the perspective of whether they are only children, there is no difference between the factors. From the perspective of the student origins, the factors of somatization, interpersonal relationships, depression, anxiety, and paranoid factors are different. There are differences between them, the mental health score of students from rural areas is higher than that of urban students, see [Table 2] for details.

(3) Mental health multi-factor analysis of university students with left-behind experience

Take the total score of the SCL-90 scale as the dependent variable (definitely, the total score of the factor is more than 160 points, the definition is positive for psychological problems, the value is 1 .

Table 1. Comparison of scores of various factors between genders

\begin{tabular}{|c|c|c|c|}
\hline Factors & $\operatorname{Boys}(\bar{X} \pm S)$ & $\operatorname{Girls}(\bar{X} \pm S)$ & $t$ \\
\hline Somatization & $1.36 \pm 0.58$ & $1.14 \pm 0.33$ & $6.953^{*}$ \\
\hline Obsessive-compulsive disorder & $1.87 \pm 0.72$ & $1.75 \pm 0.58$ & $4.733^{*}$ \\
\hline Interpersonal relationship & $1.73 \pm 0.69$ & $1.57 \pm 0.54$ & $3.907^{* *}$ \\
\hline Depression & $1.62 \pm 0.68$ & $1.54 \pm 0.51$ & $4.175^{* *}$ \\
\hline Anxiety & $1.56 \pm 0.66$ & $1.45 \pm 0.48$ & $5.125^{*}$ \\
\hline Hostility & $1.61 \pm 0.69$ & $1.47 \pm 0.53$ & $4.275^{*}$ \\
\hline Terror & $1.54 \pm 0.69$ & $1.43 \pm 0.48$ & $4.873^{*}$ \\
\hline Paranoid & $1.64 \pm 0.67$ & $1.48 \pm 0.46$ & $4.797^{* *}$ \\
\hline Psychotic & $1.52 \pm 0.69$ & $1.38 \pm 0.45$ & $5.183^{*}$ \\
\hline Other & $1.62 \pm 0.67$ & $1.45 \pm 0.47$ & $4.773^{* *}$ \\
\hline$* *:(p<0.01), *:(p<0.05)$ & &
\end{tabular}

Table 2. Comparison of scores of student factors between student origin area

\begin{tabular}{|c|c|c|c|}
\hline Factors & $\operatorname{Rural}(\bar{X} \pm S)$ & $\operatorname{Urban}(\bar{X} \pm S)$ & $t$ \\
\hline Somatization & $1.20 \pm 0.45$ & $1.16 \pm 0.40$ & $2.405^{*}$ \\
\hline Obsessive-compulsive disorder & $1.77 \pm 0.61$ & $1.77 \pm 0.66$ & $1.161^{*}$ \\
\hline Interpersonal relationship & $1.64 \pm 0.62$ & $1.55 \pm 0.56$ & $2.241^{*}$ \\
\hline Depression & $1.55 \pm 0.57$ & $1.52 \pm 0.54$ & $2.377^{* *}$ \\
\hline Anxiety & $1.47 \pm 0.58$ & $1.42 \pm 0.52$ & $2.164^{*}$ \\
\hline Hostility & $1.55 \pm 0.57$ & $1.51 \pm 0.57$ & $1.367^{* *}$ \\
\hline Terror & $1.46 \pm 0.55$ & $1.41 \pm 0.54$ & $1.616^{*}$ \\
\hline Paranoid & $1.58 \pm 0.54$ & $1.43 \pm 056$ & $2.026^{* *}$ \\
\hline Psychotic & $1.44 \pm 0.57$ & $1.23 \pm 0.52$ & $1.433^{*}$ \\
\hline Other & $1.50 \pm 0.57$ & $1.47 \pm 0.58$ & $0.819^{* *}$ \\
\hline$* *:(p<0.01), *:(p<0.05)$ & &
\end{tabular}

And the total score of the factor is less than 160 points, the definition is negative for the psychological problems, With a value of 0), using demographic characteristics variables, leftbehind background items, coercion factors, interpersonal relationship factors, and hostile factors as independent variables, a series of treatments were performed according to the requirements of model analysis (Combination and assignment of categorical items in categorical attribute variables, discretization of numerical variables), the forward selection method is used to filter the independent variables $\left(F_{\text {in }}=0.05\right)$ to construct a logistic regression 
model, and finally, 4 variables are retained in the model, which are teacher-student relationship, compulsion factor, interpersonal relationship factor, and hostility factor. From the standardized regression coefficients, we can know that the order of the influence of these variables on the total score of mental health of university students who have left behind is interpersonal relationship factor, hostile factor, compulsive factor, and the teacher-student relationship. Likelihood ratio test $G=93.287, P<0.05$, the model has statistical significance [Table 3].

Table 3. Logistic regression analysis of mental health of university students with left-behind experience

\begin{tabular}{|c|c|c|c|c|}
\hline Variable & $\begin{array}{c}\text { Standardized regression } \\
\text { coefficient }\end{array}$ & $\begin{array}{c}\text { Regression } \\
\text { coefficient }\end{array}$ & Wald $\chi 2$ & OR \\
\hline Constant & -4.532 & -7.771 & 17.670 & \\
\hline $\begin{array}{c}\text { Teacher-student } \\
\text { relationship }\end{array}$ & 0.860 & 1.214 & 6.894 & 2.618 \\
\hline $\begin{array}{c}\text { Obsessive-compulsive } \\
\text { disorder }\end{array}$ & 1.431 & 2.640 & 15.357 & 10.234 \\
\hline $\begin{array}{c}\text { Interpersonal } \\
\text { relationship }\end{array}$ & 2.167 & 4.124 & 27.436 & 24.376 \\
\hline Hostility & 1.957 & 3.094 & 18.247 & 13.152 \\
\hline
\end{tabular}

\section{Discussion}

(1) Focus on university students with left-behind experience; enhance social support and harmonious interpersonal relationships

In this study, $38.5 \%$ of students believe that their personality has changed, $66.7 \%$ of these $38.5 \%$ think that this personality change is positive, and only $33.3 \%$ think it is negative. Qualitative interviews of university students also found this result, which is different from the results of related studies. Similar studies mostly have preconceived suspicions, and more attention is paid to the negative effect of left-behind experience, and little attention is paid to its positive effect. From the perspective of emotional and social support, most students think that the students around them care more about themselves and have intimate friends around them. They often talk to friends or classmates, and $23.7 \%$ choose to talk to their parents, and $7.1 \%$ of the students choosing to solve problems by themselves. It's a great influence on subjective well-being, psychological pressure, and mental health. In response to such problems, university counsellors, teachers or parents should pay attention to correcting their emotions, guide them to take a proactive response to face negative events in life, try to avoid and reduce the backlog of bad emotions, encourage students to establish a good familyuniversity communication mechanism actively. The information about the background left behind should be further explored. Social support has the role of reducing stress and is an external resource that can be used during the stress process. University students, especially those with left-behind experience, are under the influence of psychological support and establish a complete social support network [5].

(2) Strengthen intervention to improve mental health

According to the criteria for determining the positive detection rate of SCL-90, among the left-behind population, the positive detection rate of the mental health problem among university students with left-behind experience is $35.58 \%$, which is higher than the level of domestic university students, suggesting that these students have positive symptoms. The test 
person may have a certain degree of psychological obstacles, which is higher than the level of non-left-behind university students, suggesting that "left-behind" is an important factor affecting mental health. When conducting mental health education for university students, focus on those with left-behind experience group of university students. According to the criterion that any one of the 10 factors has a score of $\geq 2$ to be positive for psychological wellbeing, among the university students with left-behind experience, the positive detection rate of 10 factors among the top 3 are interpersonal relationship sensitive factors, Obsessivecompulsive symptoms factor and "other", which is consistent with the research results.

In the analysis of influencing factors, from the perspective of gender, the differences between the scores of the 10 factors are statistically significant, and the scores of boys are higher than those of girls. Consistent with the situation of general university students, the research results and the psychological counselling results of teacher Zhang Lihua [6]. The left-behind experience has a positive effect on girls, which is due to the early maturity of girls. In the early period of staying behind, it is easier to take on the role of parents and to deal with and cope with the incident independently. We can find the freshmen and senior students' scores on somatization and compulsiveness are higher, which is related to freshmen entering the university with the new environment, and the pressure of graduates and job hunting. The self-harmony is poor. This is due to the special environment interpersonal relationships, coping styles, etc. Good interpersonal relationships and positive and flexible coping styles have a certain impact on their self-harmonious mental health. Therefore, students of different periods should be helped to change roles and adapt to the new environment and social psychology, establish good interpersonal relationships, and establish good life-practice coping styles [7][8]. The origin factor does not affect the psychology of university students with leftbehind experience. Although most of the university students with left-behind experience come from rural areas, with the acceleration of the urbanization process, the continuous improvement of the level of rural economy and education, and the gradual improvement of the education quality of rural students have little effect on the mental health differences of the group of university students who have left behind.

The results of this study show that left-behind experience is the main influencing factor of university students 'mental health. This influence is both negative and positive. This is an important finding of this study, which also reminds us that undergraduates, especially those in the process of mental health education of left-behind university students, attention should be paid to and play a positive role in the left-behind experience while attaching importance to the mental health of fragile women, special attention should be paid to the impact of left-behind experience on the mental health of boys, and the education of only children. Improve the social support network structure, take the initiative to establish a family-university communication mechanism, especially to play the role of university counselors, strengthen the mental health education of university students, pay attention to the life and mental health problems of university students with left-behind experience, and establish good trust, communication relationship.

\section{References}

[1] X. Zhao, J. Chen, M.-C. Chen, X.-L. Lv, Y.-H. Jiang, and Y.-H. Sun, "Left-behind children in rural China experience higher levels of anxiety and poorer living conditions," Acta Pædiatrica Nurturing the child, vol.103, no.6, pp.665-670, (2014) DOI: 10.1111/APA.12602

[2] D. W. Moller, "None left behind: Urban poverty, social experience, and rethinking palliative care," Journal of Palliative Medicine, vol.8, no.1, pp.17-19, (2005) DOI: 10.1089/jpm.2005.8.17 
[3] Z. Yong, J. W., and X. Yunbo, "Investigation and study on the status of the university students with leftbehind experience in China," International Journal of Education and Management Engineering, vol.2014, no.2, pp.18-28, (2014) DOI: 10.5815/ijeme.2014.02.03

[4] H. Li, "Relationship between negative life events and depression in university students with left-behind experience," Chinese Mental Health Journal, vol.31, no.4, (2017) DOI: 10.3969/j.issn.10006729.2017.04.013

[5] J. Hu, Q. Li, W. Zhao, W. Li, and M. Tang, "The analysis of psychological health of university students with guarded experience and ways to solve it," Research in Medical Education, vol.7, no.4, pp.439-448, (2008)

[6] L. Zhang, "Psychological analysis of university students with "left-behind experience"," Contemporary youth research, vol.2006, no.12, (2006)

[7] D. Gao and H. Zhang, "Analysis on the status of anxiety and depression of university students in a university," Medical Research and Education, vol.36, no.4, pp.41-46, (2019) DOI: 10.3969/j.issn.1674-490X.2019.04.008

[8] N. Li, "A study on the relationship between intimate relationship and social support and core self-evaluation of university students with left-behind experience," The science education article collects, vol.2019, no.8, pp.159-186, (2019) DOI: 10.16871/j.cnki.kjwhc.2019.08.072 
Mental Health Multivariate Analysis for University Students with Left Behind Experience

This page is empty by intention. 